\title{
Sensitivity enhancement of mixed-potential gas sensor by a series-connected sensor array
}

\author{
Takafumi Akamatsu $^{1}$, Toshio Itoh ${ }^{1}$, Woosuck Shin ${ }^{1}$ \\ ${ }^{1}$ Electroceramics Group, Inorganic Functional Materials Research Institute, \\ National Institute of Advanced Industrial Science and Technology (AIST), \\ Shimo-shidami, Moriyama-ku, Nagoya 463-8560, Japan \\ Corresponding author's e-mail address: t-akamatsu@aist.go.jp
}

\begin{abstract}
:
Mixed-potential gas sensors with an anhydrous proton conductor consisting of zinc metaphosphate glass powder and benzimidazole were fabricated. Reference and sensing electrodes of gold and platinum, respectively, were prepared on an alumina substrate, and a proton conductor was deposited between these electrodes to prepare a gas sensor. The sensor responses to 1-25 ppm $\mathrm{H}_{2}$ gas in air were investigated. Sensor array with 7 sensors connected in series showed a response to the $\mathrm{H}_{2}$ gas, even at $1 \mathrm{ppm}$. The sensitivities of the single sensor and sensor array were -21 and $-130 \mathrm{mV} / \mathrm{decade}$, respectively. By connecting 7 sensors in series, the sensor response and sensitivity were improved.
\end{abstract}

Key words: mixed-potential, gas sensor, hydrogen, phosphate glass, sensor array

\section{Introduction}

Mixed-potential gas sensors using yttriastabilized zirconia (YSZ) electrolyte have been investigated for $\mathrm{H}_{2}$ detection [1]. These gas sensors must be operated at temperatures above $500{ }^{\circ} \mathrm{C}$ to provide both satisfactory response rates against target gases and good gas selectivity. These gas sensors require a heater to maintain the operating temperature above $500{ }^{\circ} \mathrm{C}$, which results in high power consumption. Mixed-potential gas sensors that use electrolytes with high proton conductivity, such as Nafion, zirconium phosphate, and antimonic acid, have been reported to function at reduced operating temperatures [2]. However, these electrolytes must be impregnated with water to achieve high proton conductivity, which necessitates the inclusion of a complicated humidity control system to allow these sensors to operate at room temperature and under high humidity. Moreover, these gas sensors show low selectivity for $\mathrm{H}_{2}$ against $\mathrm{CO}$. One approach to overcome this challenge is to use anhydrous proton conductors at an intermediate temperature between 100 to $300{ }^{\circ} \mathrm{C}$. However, to the best of our knowledge, no mixedpotential gas sensors using a high-protonconductivity electrolyte at intermediate temperatures have been reported.
We reported a mixed-potential gas sensor using an anhydrous electrolyte consisting of zinc metaphosphate glass and benzimidazole [3]. The sensor showed good sensor response to $\mathrm{H}_{2}$ in the concentration range 250-25,000 ppm in both humid and dry air at $120{ }^{\circ} \mathrm{C}$. In this work, it was demonstrated that the sensor response could be enhanced by connecting the sensors in series.

\section{Experimental}

A glass with a nominal molar ratio of $\mathrm{ZnO}-\mathrm{P}_{2} \mathrm{O}_{5}$ was prepared by melting a batch mixture of the commercially available reagent-grade chemicals $\mathrm{ZnO}$ and $\mathrm{H}_{3} \mathrm{PO}_{4}$ in a platinum crucible at $1100{ }^{\circ} \mathrm{C}$ in air for $30 \mathrm{~min}$. The melt was poured onto an iron plate and quenched by iron pressing to make glass flakes. The glass flakes were milled using an alumina mortar and pestle to below $100 \mu \mathrm{m}$ in diameter. The glass powder was mixed with benzimidazole, and the mixture was heated at $170{ }^{\circ} \mathrm{C}$ for $12 \mathrm{~h}$ to make an electrolyte. The weight ratio of the glass powder and benzimidazole was 1:3.

The electrolyte was deposited on an alumina substrate $(5 \times 15 \mathrm{~mm})$ with platinum $(\mathrm{Pt})$ and gold $(\mathrm{Au})$ electrodes, which have a $1 \mathrm{~mm}$ electrode gap and a $1 \mathrm{~mm}$ electrode width. The electrolyte-deposited substrate was heated from a back face by a hotplate, and then an electrolyte membrane was formed on the 
substrate. We obtained the single sensor and sensor array with 7 sensors connected in series, as shown in Fig. 1.

The sensor element was placed in a test chamber heated to $120^{\circ} \mathrm{C}$ in an electrical tube furnace. Synthetic dry air (80 vol\% $\mathrm{N}_{2}, 20 \mathrm{vol} \%$ $\mathrm{O}_{2}$ ) was introduced into the chamber for $10 \mathrm{~min}$, and a gas mixture of $\mathrm{H}_{2}$ in dry air was then injected for $10 \mathrm{~min}$. Subsequently, the gas mixture flow was halted and replaced injected at a flow rate of $200 \mathrm{~mL} / \mathrm{min}$. The $\mathrm{H}_{2}$ gas concentration was controlled to the values of 1 , 10, 17.5, 25 ppm in dry air.

The potential difference (electromotive force, EMF) between $\mathrm{Pt}$ and $\mathrm{Au}$ electrodes of the sensor element was recorded using a Potentio/Galvano stat (VersaSTAT4; Princeton Applied Research). During the potential difference measurements, the Pt electrode was always connected to the positive terminal of the Potenio/Galvano stat. The EMFs of the sensor in the air and gas mixtures are denoted as $V_{a}$ and $V_{g}$, respectively. The sensor response $(\triangle E M F)$ is defined as $\triangle E M F=V_{g}-V_{a}$.

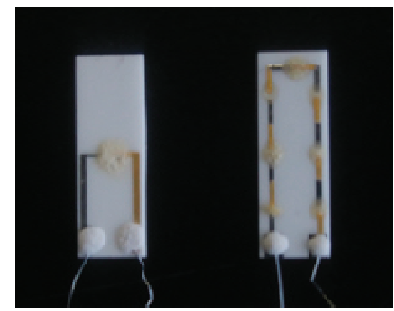

Fig. 1. Optical image of single sensor (left) and sensor array (right).

\section{Results and discussion}

Fig. 2 shows the response of the single sensor to different concentrations of $\mathrm{H}_{2}$ from $1 \mathrm{ppm}$ to $25 \mathrm{ppm}$ in air at $120^{\circ} \mathrm{C}$. No sensor response to $1 \mathrm{ppm} \mathrm{H}_{2}$ was shown. The $\triangle \mathrm{EMF}$ decreased and reached to saturation values during flowing of $\mathrm{H}_{2}$ gases for less than $5 \mathrm{~min}$. The $\triangle E M F$ increased with the $\mathrm{H}_{2}$ gas concentration.

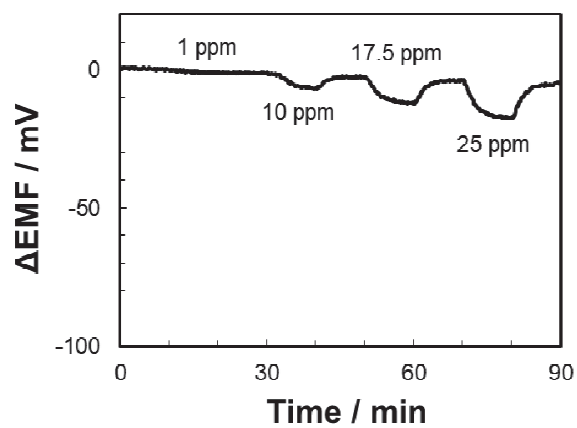

Fig. 2. Response of the single sensor to different concentrations of $\mathrm{H}_{2}$ from 1 to $25 \mathrm{ppm}$ in dry air at $120^{\circ} \mathrm{C}$.
Fig. 3 shows the response of the sensor array to different concentrations of $\mathrm{H}_{2}$ from $1 \mathrm{ppm}$ to $25 \mathrm{ppm}$ in air at $120^{\circ} \mathrm{C}$. The sensor array showed a distinct response to the $\mathrm{H}_{2}$ gas, even at 1 ppm. Fig. 4 shows relationship between sensor response and the log of the $\mathrm{H}_{2}$ concentration for single sensor and sensor array. $\triangle E M F s$ were found to be linear to the log of $\mathrm{H}_{2}$ concentration. The sensitivities of the single sensor and sensor array were -21 and $-130 \mathrm{mV} /$ decade, respectively. The sensitivity of sensor array was roughly seven times as compared to that of single sensor.

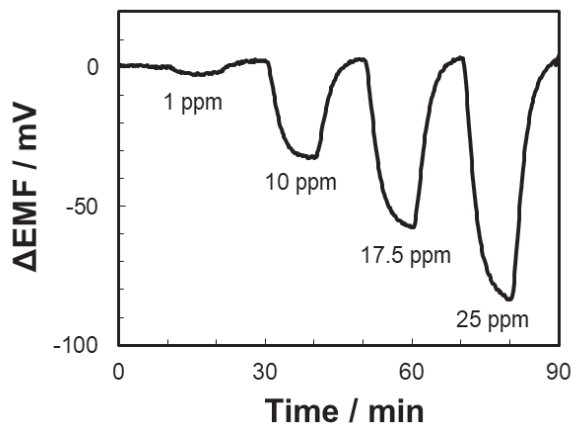

Fig. 3. Response of the sensor array to different concentrations of $\mathrm{H}_{2}$ from 1 to $25 \mathrm{ppm}$ in dry air at $120^{\circ} \mathrm{C}$.

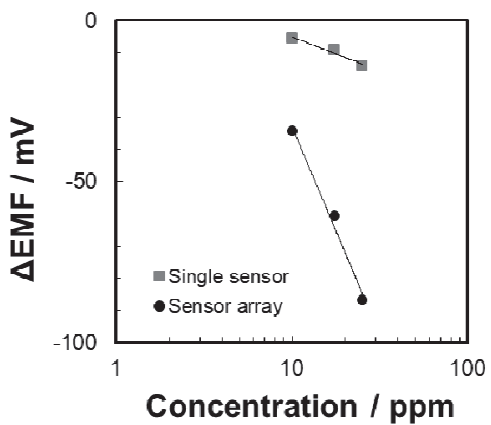

Fig. 4. Relationship between $\triangle E M F$ of single sensor and sensor array and $\mathrm{H}_{2}$ gas concentration.

\section{Acknowledgement}

This work was partially supported in part by Naito Research Grant.

\section{References}

[1] G. Lu, N. Miura, N. Yamazoe, High-temperature hydrogen sensor based on stabilized zirconia and a metal oxide electrode, Sensors and Actuators $B$ 35-36, 130-135 (1996); doi: 10.1016/S09254005(97)80042-1

[2] N. Miura, T. Harada, Y. Shimizu, T. Seiyama, A proton conductor gas sensor operative at ordinary temperature, Sensors and Actuators B 1, 125-129 (1990); 10.1016/0925-4005(90)80186-4

[3] T. Akamatsu, T. Itoh, W. Shin, Mixed-potential gas sensors using an electrolyte consisting of zinc phosphate glass and benzimidazole, Sensors 17, 97-104 (2017); doi: $10.3390 / \mathrm{s} 17010097$ 